\title{
52 EXPLORING SUSTAINABLE VIRTUAL ENTERPRISES: TWO CASE STUDIES
}

\author{
Ronald C Beckett \\ The Reinvention Network/ University of Wollongong, $\underline{r c b @ \text { reinvent.net.au }}$
}

AUSTRALIA

\begin{abstract}
From joint Industry - University research into the key success factors that sustain Virtual Enterprises, it is observed that conditions required for their successful establishment do not necessarily sustain them in the long run. Two case studies describing long-standing collaborations - one relating to a manufacturing network, and the other to a professional virtual community are presented Transactions that are valued by the participants, a balance between similarity and complementarity attributes of the participants and effective utilisation of time are seen as important sustaining factors.
\end{abstract}

\section{INTRODUCTION}

This paper considers the dynamics of participation in business networks from the viewpoint of individual SME's in an Australian context. The proportion of Australian SMEs actively involved in networks and alliances appears to be limited and the long-term success rate of business network alliances seems low. What sustains the successful ones, how might they be characterised and how have they evolved over time?

Previous work by the author and some university colleagues over the past decade has explored different kinds of business networks, mapping how they deliver benefits to participants, including mapping knowledge aspects of the business processes involved (Beckett, 2002; Beckett, 2003, Beckett, Hyland and Sloan, 2003). This experience has suggested that some factors related to time allocation and level of effort may inhibit the participation of smaller firms in formal collaborations, that their ongoing participation will depend on how the benefits of collaboration would compare with benefits from time invested in alternative opportunities, and on the dynamics of their market and technology environment. Initial research indicated that firms who did not achieve any perceived value-adding transactions from participation in a collaboration were unlikely to continue to participate, leading us to focus on value-adding transactions. In the following parts of this paper, two case studies of enduring Virtual Enterprises (VE's) are presented, and the anatomy of a successful collaboration is mapped using these observations. 


\section{CASE STUDIES}

\subsection{A Manufacturing Network}

A study of Australian Toolmaking Innovation Pty Ltd (ATI) was carried out using both a questionnaire and interviews. Both the questionnaire and the interview structure built on previous work by Fulop and Kelly (1997). ATI is a virtual enterprise acting as a shopfront for a network of toolmakers, and it has been in existence about nine years. The primary goal of the ATI network at the time of its formation was acquiring production orders from overseas. A secondary goal was to make the members stronger in the domestic market. The member firms in this network are all SMEs with between 20 and 100 employees. There are presently 5 firms in the network, all located in the southern Sydney region. Members find proximity important mainly due to the ease of communication, and secondly for inter-firm transportation of products. There is a full time manager who operates independently, but is funded by the network partners. A Board of partners provides general direction and strategy. There have been three different managers to date.

The network members describe similarities in a number of areas. Product or service: All members make the same type of tooling (predominantly plastic tooling) of high quality. Within that sector each has a speciality in their own field. Customers: The type of customer is similar for all members and the largest customers are automotive companies. Mode of operation: The members are among the larger toolmakers in the region and are regarded as very progressive in toolmaking. The mode of operation of the members is very much the same in the way that they all quote for orders, and have similar methods of production. All members are privately owned companies. Territory or market: ATI operates in two distinctive types of market, the domestic market (Sydney, New South Wales and Victoria) and the export market. All members also operate in the same domestic market. They have acquired their own overseas markets, although these are of a minor scale. The firms aimed to expand into the international market in collaboration using the network's capacity to win large orders. Relationships that developed between participants resulted in joint purchasing, sharing of resources and capacity to meet peak demands of long-term domestic customers, and information sharing on new technologies. These practices provided tangible, but initially unplanned benefits.

The members have some complementary capabilities. Every member has some niche specialization within their common market sector. Members can share work with each other in confidence of quality and reliability. This gives the network a wide range of services to offer in tooling. Whilst the network has reduced its internal costs, and increased revenue by sharing some domestic market projects, the level of revenue hoped for from export work was not initially achieved. However, in recent years, following deployment of the full time Network Manager to China for lengthy periods, business has improved, and the VE is profitable in its own right on the basis of export orders. The experience has lead to the partners consider joint ownership of assets and new business ventures. 


\subsection{A Professional Virtual Community}

This network was studied using a questionnaire as a checklist to adopt a consistent approach to individual interviews. It is an informal innovation network of industrial designers, engineers, tool designers and tool manufacturers tending to specialise in the development of unique plastic product designs that might also require the development of unique production processes. Activities commenced in 1986 with just a few companies, and there are now about 15 participants distributed across five States in Australia. Each participant has less than ten employees, and the main criteria for participation seems to be demonstrated excellence in a specialisation, ease of collaboration and demonstrated contractually compliant performance. New business opportunities may be identified by any member who may then take the lead position for that project, or an opportunity may be referred to a more appropriately specialised partner for follow-up and leadership. The network arrangement enables members to offer turnkey product design and tooling packages to clients rather than just part of a package. The maintenance of a reputation for innovation and reliability is an important consideration for each individual participant, and in taking a lead position, they become interdependent on the others to maintain that reputation. The network itself is not a visible entity. Membership has changed over time as more specializations have been added, and as difficulties have arisen with either ease of collaboration or contract compliance with past members.

One of the members has a strong orientation towards collaborative arrangements arising from prior experience in Europe and appears to be the network champion, as he sets standards and protocols (eg everyone uses Microsoft Project software for project management). Although the network structure is informal, individual projects are formally operated with clearly defined roles and tasks. This network is based on accessing and sharing intellectual assets rather than physical assets, and it is in place to expand the market access of the participants rather than to share knowledge. Participants have noted some expansion of personal networks as a benefit. Technology intelligence and operational intelligence are seen as the main beneficial knowledge transactions. Some members of the network are considering establishing a more formal arrangement to develop their own products and brand.

\section{SUSTAINING COLLABORATIONS}

In these two case studies the collaboration motivations were similar: to engage in a broader market. In one case a formal company structure was established, and this drove some of the internal business processes. In the other case, an informal network has been retained, with undocumented rules about behaviour relating to trust. Two distinctive phases in the evolution of these VE's have been observed: an establishment phase and a sustaining phase

\subsection{Establishing a Virtual Enterprise}

Resources must be made available when entering into any collaboration, and these resources need to be continually invested in organising and maintaining the 
relationships. One of the network partners interviewed characterised this as naivety and persistence. There are views that a certain minimum resource base is required to establish an effective network, and a survey on innovation networks by Basri, (2001) suggests a company size of at least ten employees is needed for effective participation. And yet in the professional network example presented here, smaller companies have had enduring relationships. Consistent with the observations of Marceau (1999) it is argued that there also needs to be business activities that improve the market positioning for the partners involved in the alliance if the alliance is to be ongoing. Just establishing a collaboration framework is not enough, there must be both business and housekeeping activities and benefits for it to endure.

\section{Internal operations}

Considered from an operational perspective, there are many forms of collaboration being practiced today, albeit by a minority of firms, ranging from one-on-one alliances through to multi-firm industrial clusters. But they all have to confront a number of common issues, such as:

- Integration. For alliances to be effective, certain unique practices need to be developed and embedded in the partner organisations. Some forms of VE business systems must be agreed upon. Industry norms provide this to some extent in the two case examples.

- Organisational barriers. Most Firms have developed a repetoire of practices that help make them unique, so they restrict access to these practices and maintain an independent management style. But collaboration requires sharing or changing of some established practices and the acceptance of a degree of interdependency on other Firms, as well as the development of a common language/jargon.

- Trust. Compared to 'simple' inter-firm customer-supplier relationships, knowledge exchange requires each of the partners to allow the other partners to look into each other's operations. In addition, priorities given to internal work and work associated with the collaboration must be equitably balanced. As this cannot be enforced upon any of the partners, an above average level of trust in and respect for each other's competencies and best intentions is required. This is seen as a prerequisite in the two cases.

\section{External engagement}

Marceau (1999) observed that of several hundred network arrangements stimulated with Australian Government support in the mid 1990's, some worked very well and others had not conducted a common project after several years. She noted that whilst considerable attention had been paid to business planning and the internal functioning of these networks, competencies in these areas did not seem to assure the long-term viability of a network. Marceau $(1999,23)$ saw that the "success of collaborative ventures lies in the organisation of the external environment rather than the climate internal to the network", She discussed the external environment in terms of the rate of technology change, market growth opportunities and opportunities to access markets. Both case examples are about accessing market opportunities. 


\subsection{Sustaining a Virtual Enterprise}

Given that internal arrangements and external engagement have been established, the following attributes have been observed in collaborations that endure.

\section{Balancing similarity and complementarity}

Enterprises may choose to collaborate with others similar to them (eg all toolmakers in case example one), generally to expand scale, or with others complementary to them (eg toolmakers, product designers and engineers in case example two), generally to expand scope. Each of these arrangements puts different demands on a collaboration, and in the long run a blend of similarity and complementarity emerges. Otherwise the similar enterprises will remain arms length competitors, and the complementary enterprises will have insufficient common focus.

\section{Delivering value}

In an industrial network each firm possesses different capabilities and knowledge and through both cooperation and competition, according to Foss (1999) the network is able to effectively bundle and use these capabilities in ways that a single firm is unable to. Similarly Ernst and Kim (2001) maintain that industrial networks act as a catalyst for knowledge diffusion and capability formation. Simmie et al (2002) describe how industrial networks as centres of innovation can be considered to provide several benefits to participating firms. They maintain that small firms in a network can act as closely knit production unit, and they facilitate local supply chains so reducing transaction costs and taking advantage of a skilled labour pool that they do not have to pay for directly. Within the network the movement of labour amonst firms can create a learning system that transfers knowledge between firms. Here we see both economic and knowledge access factors in play, as observed in the two cases.

\section{Leveraging time}

Whilst a collaboration will enable a particular participant to access additional resources and knowledge with little monetary expenditure, significant amounts of time must be invested in collaborations to extract full value. There is clearly some up-front time to establish a collaboration, but time must also be continuously invested for it to endure. This can be a difficulty for an SME unless time is used efficiently, and unless the time invested enhances the capability and the capacity of the collaboration partners.

Participant commitment to a collaboration is seen as an important success factor by some observers. However some experienced industry practitioners suggest that only $20 \%$ or $30 \%$ of an enterprise's resources should be committed to a particular collaboration as a means of minimising the impact of possible failure. The idea of limited participation also makes sense from another perspective too. A strong team is made up of strong players, and the primary focus of participants must remain on effective operation of their own enterprise. Excellence was seen as a prerequisite for participation in the two cases presented. 


\section{A Focus on Transactions}

Transactions between participants are the lifeblood of a collaboration. No transactions, no collaboration. Some transactions of a housekeeping nature are necessary to sustain effective operations, as are some transactions related to the management of tasks and relationships. But there must also be transactions that deliver value to the participants and their clients; otherwise the collaboration will not endure (Beckett, Hyland and Sloan, 2003).

\section{Housekeeping transactions}

There is a variety of housekeeping transactions needed to sustain a collaboration, along with some assignment of responsibility for managing these transactions. Transactions associated with managing relationships, transactions associated with managing tasks and transactions associated with maintaining communication are observed at work. In making broadly similar observations, some researchers see that generic roles need to be enacted. Zhou (2001) describes three roles: a coordinator function to manage the enterprise identity, capabilities, capacity and performance; a collaborator function that facilitates knowledge, information exchange and intercompany relationships; and a communicator function that facilitates face-to-face or virtual meetings and keeps all participants aware of the status of the collaboration. Lipnack and Stamps (2000) have noted five leadership roles that address interfacing needs in the evolution of technology enabled virtual teams: coordinator, designer, disseminator, Tech-net manager, Socio-net manager and executive champion. These roles have both operational and political dimensions.

\section{Value adding transactions}

Previous work (Beckett, Hyland and Sloan, 2003) has shown there are three kinds of transactions that are valued by VE participants: Those that build economic capital, those that build social capital, and those that build knowledge capital. Direct business transactions might enhance revenue, reduce costs, optimise the use of assets, reduce lead-time, enhance reliability or reduce risk. Biggiero and Sammarra (2001) point out that sustainable inter-organisation networks are not only driven by current economic motivations, but by some socio-psychological factors that may lead to future cooperative relationships, and this has been observed in the two cases presented above. Social transactions might enhance an organisation's power base, expand personal networks, reduce perceived inter-organisational risk by better understanding the motives of participants, enhance business environment understanding, or provide an opportunity to test ideas in a friendly environment. Wiendahl, Engelbrecht and Hamacher (2001) see cooperation to meet production demands and learning relationships that support market demands as increasingly important. Knowledge transactions might provide market intelligence, technology intelligence, and operational intelligence, reduce decision-making risk by seeing where new concepts have been successful, and exercise organisational learning competencies that help sustain change capacity in a dynamic environment. Some transactions may provide benefits now, and others may provide future benefits. 


\section{THE ANATOMY OF A SUSTAINABLE COLLABORATION}

From the observations presented in the previous section of this document, a form of map has been produced that summarises the key activities involved in establishing and in sustaining a collaboration, and the linkages between the various activities. This is shown in Figure 1, which has proven to be a useful framework for discussing issues of collaboration and virtual enterprises in industry forums.
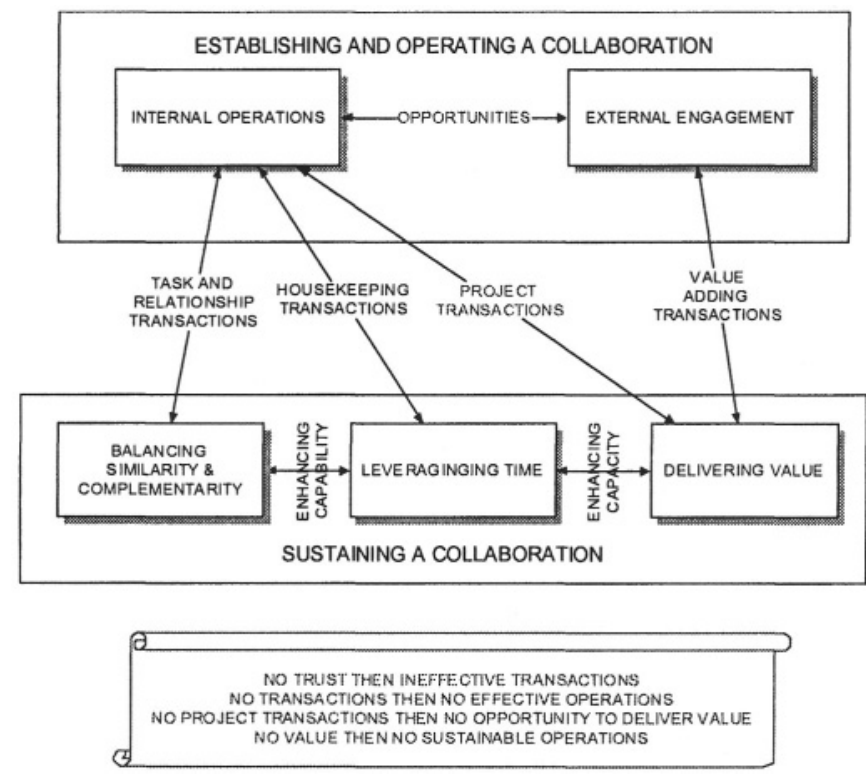

Figure 1: A Sustainable Collaboration Activity Map

\section{CONCLUDING REMARKS}

Two generic activities required to establish an effective Virtual Enterprise have been suggested: one establishing internal protocols, and another concerned with external engagement, with specific opportunities for collaboration providing a link between them. Three additional activities are suggested as necessary for sustaining such a Virtual Enterprise collaboration. The first is an activity that delivers perceived value by building economic, knowledge or social capital to enhance capacity. The second is an activity that balances participant similarity and complementarity attributes to enhance their collective capability. The third activity involves leveraging the time of the participants to maintain a balance between effective operations in their own enterprises, servicing VE project requirements, and expanding the capabilities of the VE. It is observed that this third activity may be a limiting factor for small companies, and does not appear to be comprehensively researched. The three sustaining activities are linked to the establishment ones by a number of different 
kinds of transactions needed for effective operations. The Generic activity "map" presented here is being used by the author to help SME's design more effective collaborations.

\section{REFERENCES}

1. Basri, E. (2001) "Inter-firm Technological Collaboration in Australia in an International Context: Implications for Innovation Performance and Public Policy" in "Innovation Networks: Cooperation in National Innovation Systems", OECD, pp 143-168

2. Ronald C Beckett (2002) "The evolution of an Aerospace innovation network: a ten year case study" - in Proceedings of the IMP Group, Asia Conference "Culture and Collaboration in Distribution Networks", Perth, Australia Dec 11-13, 2002, ISBN 1740671848

3. Beckett, R.C; Hyland, P and Sloan, T (2003) "Mapping Collaborative Transactions in Networks that Yield Business Benefits" Proc $19^{\text {th }}$ Annual IMP Conference, University of Lugano, Switzerland, 4-6 September (ISBN 82-7042-576-1)

4. Ronald C Beckett (2003) "Determining the anatomy of business systems for a virtual enterprise" Computers in Industry, Vol 51, Issue 2, June pp 127-138

5. Biggiero, L. and Sammarra, A., (2001) 'Similarity and complementarity in inter-organisational networks' APROS (Asia-Pacific Researcher in Organisation Studies), Hong Kong Baptist University, 3-5 December

6. Ernst, D. and Kim, L. (2001) Global Production Networks, Knowledge Diffusion and Local Capability Formation. Nelson \& Winter Conference Aalborg Denmark June 12-15

7. Foss, N. (1999) Networks, capabilities and competitive advantage. Scandinavian Journal of Management 15 1-15

8. Fulop, L and Kelly, J. (1997) "A Study of Business Networks in Australia" Sydney: Australian Business Chamber

9. Lipnack, J., and Stamps, J., (2000) Virtual teams: people working across boundaries with technology John Wiley \& Sons, New York

10. Marceau, J., (1999) 'Networks of innovation, networks of production and networks of marketing: collaboration and competition in the biomedical and toolmaking industries in Australia' Creativity and Innovation Management, Vol 8, No 1, March, pp20-27

11. Simmie, J., Sennett, J., Wood, P. and Hart, D. (2002) Innovation in Europe: A Tale of Networks, Knowledge and Trade in Five Cities. Regional Studies, vol 36, No 1 pp 47-64

12. Wiendahl, E.H, Englebrecht, A and Hamacher, O (2001) "From Single Enterprises to Complementary Networks" In Mo, J.P.T and Nemes, L (Eds) "Global Engineering, Manufacturing and Enterprise Networks", Kluwer Academic Publishers, The Netherlands, pp 66-73

13. Zhou, M (2001) "The architecture of an internet-based Virtual Industrial Community" In Mo, J.P.T and Nemes, L (Eds) "Global Engineering, Manufacturing and Enterprise Networks", Kluwer Academic Publishers, The Netherlands, pp61-65 